Int. J. Odontostomat., 8(2):215-220, 2014.

\title{
Las Revistas Odontológicas en la Base SciELO: Una Mirada Bibliométrica
}

\author{
Dental Journals in the SciELO Database: A Bibliometric Overview
}

Mario Cantín ${ }^{* * *, * * *} \&$ Yanela Aravena ${ }^{* * *}$

CANTíN, M. \& ARAVENA, Y. Las revistas odontológicas en la base SciELO: una mirada bibliométrica. Int. J. Odontostomat., 8(2):215-220, 2014.

RESUMEN: Las revistas desempeñan un importante rol en el proceso de comunicación científica para diversas ciencias, incluyendo a la Odontología. Dicha comunicación adquiere mayor relevancia si las revistas están indexadas en una base de datos reconocida nacional e internacionalmente. El objetivo de esta investigación fue buscar, analizar y describir las revistas de odontología de indexadas en el registro completo de países en la base SciELO hasta el primer semestre del año 2014, con el fin de dar a conocer a la comunidad odontológica su estado y características principales. Se diseño un estudio bibliométrico descriptivo, donde se identificaron las revistas odontológicas en las base SciELO, abreviación, fecha de admisión de cada revista en la colección, números disponibles, cantidad y tipo de artículos e idioma de publicación. Se identificaron 17 revistas odontológicas indexadas en la base SciELO, siendo Brasil quien posee más revistas, seguido de España, Chile y Colombia. La primera revista ingresó el año 2000, y el 2014 hubieron 2 nuevos ingresos. Hubo una relación directa entre el año de ingreso y cantidad de números publicados. Hasta la fecha un total de 5.684 artículos se han publicado publicado, principalmente artículos originales, reportes de caso, revisiones de la literatura, editoriales y cartas al editor. El idioma más utilizado para publicar fue el inglés $(55,2 \%)$, seguido del español $(31,4 \%)$. El idioma portugués, sólo representó el 2,3\%. En algunas revistas, se observó la publicación simultánea inglés/español e inglés/portugués (3,8\%). La odontología, y sus revistas temáticas, han podido integrarse de manera exitosa a los diversos componentes de la metodología SciELO. Es necesario que los odontólogos conozcan las revistas disponibles en SciELO y sus características como el números de artículos e idioma de publicación, periodicidad, entre otros, con el fin realizar una selección adecuda al momento de enviar su publicación.

PALABRAS CLAVE: publicación científica, revistas, odontología, base de datos, SciELO.

\section{INTRODUCCIÓN}

La comunicación científica se realiza, tradicionalmente, a través de la publicación de artículos en revistas especializadas, quienes constituyen el vehículo por excelencia de la comunicación del conocimiento científico. Por este motivo, el estudio de las revistas, publicaciones de un centro, área, comunidad o país, son indicadores válidos para cuantificar la actividad científica (Pérez, 2001; Tomás-Casterá et al., 2010).

Las revistas científicas desempeñan un importante rol en el proceso de comunicación científica, ya que constituyen la principal vía para la validación del nuevo conocimiento y hacen posible su difusión dentro de la comunidad de profesionales e investigado- res, siendo el principal medio de comunicación para diversas áreas científicas, entre ellas la odontológica.

La utilidad del análisis de las revistas y publicaciones para el estudio de la actividad investigadora ha llevado a la bibliometría a experimentar un gran desarrollo. Estos trabajos permiten estar al tanto y valorar la producción científica de un área de conocimiento y entre sus principales aplicaciones se encuentra el área de la política científica, siendo además utilizados en los procesos de evaluación de los resultados de la actividad investigadora. Para esto, es necesario que los indicadores de producción, al igual que el resto de los indicadores de Ciencia y Tecnología, sean recopila-

\footnotetext{
* Centro de Investigación CIMA, Facultad de Odontología, Universidad de La Frontera, Temuco, Chile.

* Doctorado en Ciencias Morfológicas, CEMyQ, Facultad de Medicina, Universidad de La Frontera, Temuco, Chile.

*** Centro de Investigación en Ciencias Biomédicas, Universidad Autónoma de Chile, Temuco, Chile.

${ }_{* * * *}^{*}$ Estudiante. Escuela de Odontología, Facultad de Odontología, Universidad de La Frontera, Chile.
} 
dos con una metodología común, generalmente aceptada, para que sus resultados puedan ser comparados y comparables (Tomás-Casterá et al.).

Así, el seguimiento y análisis de una publicación periódica a través de indicadores bibliométricos permite obtener unos resultados objetivos de gran interés, así como observar las tendencias que siguen las revistas. Los datos que obtienen y las conclusiones que se deducen pueden ayudar a los odontólogos e investigadores en ciencias odontológicas a elegir de manera adecuada una revista, así como a los responsables de las revistas, a mejorar la gestión de la misma, facilitando la toma de decisiones en aspectos relacionados, por ejemplo, con la selección de artículos (Granda-Orive et al., 2002; Tomás-Casterá et al.).

El objetivo de esta investigación fue buscar, analizar y describir mediante el análisis bibliométrico, las revistas de odontología de indexadas en la base SciELO hasta el primer semestre del año 2014.

\section{MATERIAL Y MÉTODO}

Se realizó un estudio bibliométrico descriptivo de corte transversal. La búsqueda incluyó a todas las revistas del área odontológica indexadas en la base SciELO (Scientific Electronic Library Online), hasta el mes Junio del año 2014, con disponibilidad en idioma español. Se excluyeron revistas que no presentaran información online disponible, sin acceso o que fueran eliminadas de la base de datos.

La búsqueda se realizó desde el portal web de SciELO (http://www.scielo.org/php/index.php), a fin de obtener acceso a toda la red, a través de la sección "colecciones de revistas", donde se encuentran los links de acceso para cada país (Argentina, Brasil, Chile, Costa Rica, Cuba, España, México, Perú, Portugal, Sudáfrica y Venezuela), junto a los países en desarrollo (Bolivia, Paraguay y Uruguay).

En el sitio SciELO de cada país, fue seleccionada la "Lista por materia" y luego el ítem "Ciencias de la salud" el cual mostró los títulos de las revistas relacionadas. Fueron seleccionados los títulos que se relacionaran con el área odontológica, con el fin acceder a su página e información básica. Si esta información explicitaba la publicación de artículos relacionados con odontología y sus diversas áreas, fue considerada como revista odontológica e ingresó al estudio. Además, se obtuvo la abreviación de cada revista seleccionada, ISSN y el tipo de artículos que publica.

También, desde la página principal de la revista, se seleccionó el ítem "instrucciones a los autores", apartado "alcance y política editorial" para obtener información correspondiente a los idiomas aceptados por cada revista.

Para obtener los números disponibles y fecha de admisión en SciELO de cada revista, se seleccionaron todos los números. Se cuantificaron los artículos en cada número disponible desde la "tabla de contenidos" que muestra los títulos publicados.

Finalmente, para determinar la proporción de los idiomas aceptados en las publicaciones de cada revista, se contabilizaron los artículos realizados desde la "tabla de contenidos" de cada número emitido hasta el primer semestre del año 2014. Desde esta sección se cuantificaron y clasificaro los tipos de artículos publicados. Los datos recopilados fueron ingresados a tablas de doble entrada, y la información fue analizada mediante estadística descriptiva.

\section{RESULTADOS}

Se identificaron 17 revistas odontológicas indexadas en la base SciELO durante el periodo 2014 (primer semestre). Al analizar el número de revistas según los países que integran la red SciELO, Brasil posee 6 revistas, España 3 revistas, Chile y Colombia 2 revistas cada uno y con sólo 1 revista Cuba, México, Sudáfrica y Uruguay. No se encontraron registros para Argentina, Bolivia, Costa Rica, Paraguay, Perú, Portugal y Venezuela. Junto a ellas, en la red SciELO Colombia, se encontró una revista (Revista de la Universidad Industrial de Santander. Salud) que no presenta una misión explícita o única vinculada a la odontología, pero los odontólogos son junto a otros profesionales de la salud parte de su audiencia o público objetivo. Todas las revistas presentan títulos abreviados e ISSN. La mayoría posee registro Impreso y on-line, a excepción de la Revista Odontológica Mexicana con registro únicamente impreso y la South African Dental Journal sólo electrónico (Tabla I). Ocho revistas modificaron su nombre previo ingreso a SciELO (Tabla II).

De acuerdo a la fecha de admisión en SciELO, los años 2000 y 2001 ingresó una revista de España 
Tabla I. Revistas odontológicas indexadas en SciELO el año 2014 (primer semestre) pertenecientes a países que integran la red SciELO y su registro ISSN.

\begin{tabular}{|c|c|c|c|c|}
\hline \multirow{2}{*}{ País } & \multirow{2}{*}{ Título Completo } & \multirow[t]{2}{*}{ Título abreviado } & \multicolumn{2}{|c|}{ ISSN } \\
\hline & & & Online & Impreso \\
\hline Argentina & --- & --- & --- & --- \\
\hline \multirow{6}{*}{ Brasil } & Brazilian Dental Journal & Braz. Dent. J. & $1806-4760$ & 0103-6440 \\
\hline & Brazilian Journal of Oral Sciences & Braz. J. Oral Sci. & $1677-3225$ & $1677-3217$ \\
\hline & Brazilian Oral Research & Braz. Oral Res. & $1807-3107$ & $1806-8324$ \\
\hline & Dental Press Journal of Orthodontics & Dental Press J. Orthod. & $2177-6709$ & $2176-9451$ \\
\hline & Journal Of Applied Oral Science & J. Appl. Oral Sci. & $1678-7765$ & $1678-7757$ \\
\hline & Revista de Odontología da UNESP & Rev. Odontol. UNESP & $1807-2577$ & 0101-1774 \\
\hline Bolivia & --- & --- & --- & --- \\
\hline \multirow[t]{3}{*}{ Chile } & International Journal of Odontostomatology & Int. J. Odontostomat. & 0718-381X & 0718-3801 \\
\hline & Revista Clínica de Periodoncia, Implantología y & Rev. Clin. Periodoncia & & \\
\hline & Rehabilitación Oral (PIRO) & Implantol. Rehabil. Oral & 0719-0107 & $0718-5391$ \\
\hline \multirow[t]{3}{*}{ Colombia } & CES Odontología & CES Odontol. & $0120-971 X$ & $0120-971 X$ \\
\hline & $\begin{array}{l}\text { Revista Facultad de Odontología Universidad } \\
\text { de Antioquia }\end{array}$ & Rev. Fac. Odontol. Univ. Antiog. & $2145-7670$ & $0121-246 x$ \\
\hline & $\begin{array}{l}\text { Revista de la Universidad Industrial de } \\
\text { Santander. Salud }\end{array}$ & $\begin{array}{l}\text { Rev. Univ. Ind. Santander. } \\
\text { Salud }\end{array}$ & $2145-8464$ & 0121-0807 \\
\hline Costa Rica & --- & --- & --- & --- \\
\hline Cuba & Revista Cubana de Estomatología & Rev. Cubana Estomatol. & $1561-297 X$ & 0034-7507 \\
\hline \multirow[t]{3}{*}{ España } & Avances en Odontoestomatología & Av. Odontoestomatol. & $2340-3152$ & 0213-1285 \\
\hline & Avances en Periodoncia e Implantología Oral & Av. Periodoncia. & 2340-3209 & $1699-6585$ \\
\hline & Revista Española de Cirugía Oral y Maxilofacial & Rev. Esp. Cir. Oral Maxilofac. & $2173-9161$ & $1130-0558$ \\
\hline México & Revista Odontológica Mexicana & Rev. Odont. Mex. & --- & $1870-199 x$ \\
\hline Paraguay & --- & --- - & --- & --- \\
\hline Perú & --- & --- & --- & --- \\
\hline Portugal & --- & --- & --- & --- \\
\hline Sudáfrica & South African Dental Journal & S. Afr. Dent. J. & 0375-1562 & --- \\
\hline Uruguay & Odontoestomatología & Odontoe stomatología & $0797-0374$ & $1688-9339$ \\
\hline Venezuela & -- & -- & --- & --- \\
\hline
\end{tabular}

Tabla II. Títulos de revistas odontológica indexadas en SciELO, que han modificado su nombre previo ingreso a SCIELO.

\begin{tabular}{ll}
\hline \multicolumn{1}{c}{ Título actual } & \multicolumn{1}{c}{ Titulo anterior } \\
\hline Brazilian Oral Research & Pesquisa Odontológica Brasileira \\
Dental Press Journal of Orthodontics & Revista Dental Press de Ortodontia e Ortopedia Facial \\
Journal Of Applied Oral Science & Revista Da Faculdade De Odontologia De Bauru \\
Avances en Periodoncia e Implantología Oral & Avances en Periodoncia \\
Revista Española de Cirugía Oral y Maxilofacial & Revista Iberoamericana de Cirugía Oral y Maxilofacial \\
Revista Odontológica Mexicana & Revista de la División de Estudios de Posgrado e Investigación \\
South African Dental Journal & Journal of the Dental Association of South Africa / Tydskrif van \\
& die Tandheelkundige Vereniging van Suid-Afrika
\end{tabular}

y de Cuba, respectivamente. El año 2003, el ingreso aumentó a tres revistas provenientes de Brasil (1) y España (2), mientras que el 2004, se admitieron sólo dos desde Brasil. No se observan ingresos hasta el año 2010 con dos revistas provenientes de Brasil y Colombia. El año 2011 fueron admitidas tres revista desde Chile (2), Uruguay (1), al igual que el 2013 desde México (1) y Brasil, que sumó dos nuevas revistas. Durante el 2014 (hasta el mes de Junio) hay dos nuevas revistas, desde Colombia y Sudáfrica; este último país recientemente incluido en la red SciELO.

La cantidad de números publicados se relaciona con la fecha de admisión en la red SciELO. De acuerdo a esto, las revistas con mayor cantidad de números son Avances en Odontoestomatología (67), Journal Of Applied Oral Science (62), Revista Cubana de Estomatología (60), admitidas los años 2003, 
Tabla III. Tipos y número de artículos publicados en SciELO por revistas odontológicas durante el primer semestre 2014.

\begin{tabular}{|c|c|c|c|c|c|c|c|c|c|}
\hline & AO & $\mathbf{R L}$ & RC & CE & Ed & EA & CB & $\mathbf{0}$ & Tota \\
\hline Braz. Dent. J. & 840 & 0 & 0 & 0 & 2 & 0 & 0 & 0 & 842 \\
\hline Braz. J. Oral Sci. & 77 & 3 & 10 & 0 & 0 & 0 & 0 & 0 & 90 \\
\hline Braz. Oral Res. & 700 & 2 & 0 & 0 & 51 & 0 & 0 & 0 & 753 \\
\hline Dental Press J. Orthod. & 337 & 6 & 30 & 0 & 28 & 0 & 0 & 112 & 513 \\
\hline J. Appl. Oral Sci. & 913 & 38 & 32 & 0 & 58 & 0 & 0 & 0 & 1041 \\
\hline Rev. Odontol. UNESP & 90 & 14 & 4 & 0 & 0 & 0 & 0 & 0 & 108 \\
\hline Int. J. Odontostomat. & 154 & 15 & 44 & 0 & 1 & 0 & 0 & 0 & 214 \\
\hline Rev. Clin. Periodoncia Implantol. Rehabil. Oral & 71 & 17 & 14 & 4 & 0 & 0 & 0 & 0 & 106 \\
\hline CES Odontol. & 21 & 13 & 10 & 0 & 4 & 0 & 0 & 0 & 48 \\
\hline Rev. Fac. Odontol. Univ. Antioq. & 84 & 19 & 7 & 0 & 11 & 3 & 0 & 0 & 124 \\
\hline Rev. Cubana Estomatol. & 324 & 85 & 105 & 0 & 18 & 0 & 1 & 58 & 591 \\
\hline Av. Odontoestomatol. & 287 & 0 & 0 & 0 & 0 & 0 & 0 & 68 & 355 \\
\hline Av. Periodoncia. & 181 & 0 & 1 & 0 & 0 & 0 & 0 & 44 & 226 \\
\hline Rev. Esp. Cir. Oral Maxilofac. & 139 & 64 & 124 & 18 & 17 & 0 & 0 & 181 & 543 \\
\hline Rev. Odont. Mex. & 19 & 1 & 20 & 0 & 5 & 0 & 0 & 0 & 45 \\
\hline S. Afr. Dent. J. & 0 & 3 & 1 & 1 & 1 & 0 & 0 & 7 & 13 \\
\hline Odontoestomatología & 25 & 3 & 12 & 0 & 11 & 0 & 0 & 21 & 72 \\
\hline TOTAL & 4262 & 283 & 414 & 23 & 207 & 3 & 1 & 491 & 5684 \\
\hline
\end{tabular}

$\mathrm{AO}=$ Artículo original, $\mathrm{RL}=$ Revisión de la literatura, $\mathrm{RC}=$ Reporte de caso, $\mathrm{CE}=$ Carta al editor, $\mathrm{E}=\mathrm{Editorial}, \mathrm{EA}=\mathrm{Ensayo}$ académico, $\mathrm{CB}=$ Comunicación breve, $\mathrm{O}=$ Otros.

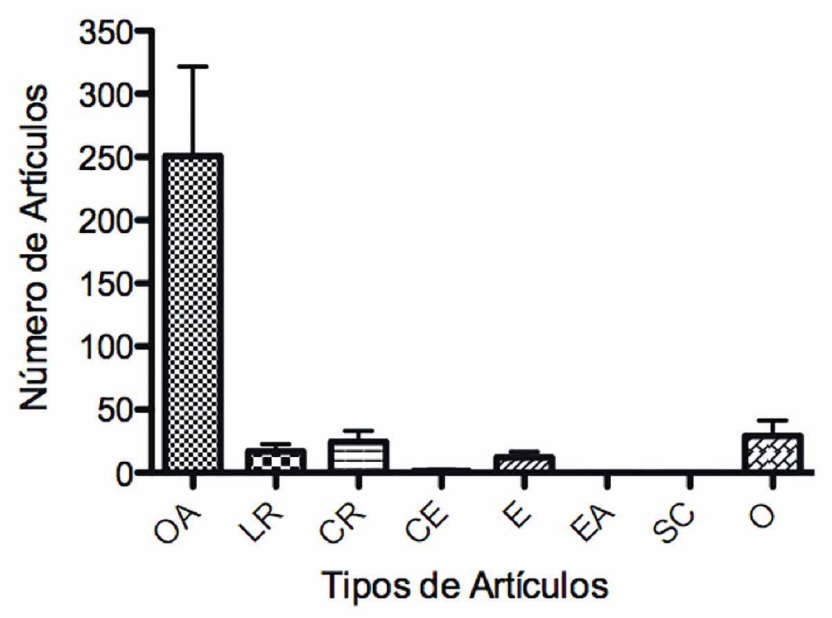

Fig. 1. Media $( \pm D E)$ de los diferentes tipos de artículos publicado en SciELO del área odontológica. $\mathrm{AO}=$ Artículo original, $\mathrm{RL}=$ Revisión de la literatura, $\mathrm{RC}=$ Reporte de caso, $\mathrm{CE}=$ Carta al editor, $\mathrm{E}=$ Editorial, $\mathrm{EA}=$ Ensayo académico, $\mathrm{SC}=$ Comunicación breve, $\mathrm{O}=$ Otros.

2001 y 2004, respectivamente. Mientras que aqueIlas que tienen menos números publicados son Brazilian Journal of Oral Sciences (6) y Revista Odontológica Mexicana (5), ingresadas el año 2013 y South African Dental Journal (1), ingresada el 2014.

El total de artículos publicados fue 5.684. El tipo de artículo más publicado corresponde a originales, seguidos de reportes de caso, revisiones de la literatura, editoriales y cartas al editor. De manera aislada, se observó la publicación de artículos tipo ensayo académico y comunicación breve. Se encontraron artículos no clasificables en los tipos anteriores, los que fueron agrupados como otros (actualizaciones, noticias, obituarios, etc.). En la Figura 1 se observa la Media $( \pm D E)$ de los diferentes tipos de artículos publicados. El detalle de los tipos de artículo según cada revista analizada se encuentra en la Tabla III.

El idioma más utilizado para publicar fue el inglés $(55,2 \%)$, seguido del español $(31,4 \%)$. El idioma portugués represento sólo el 2,3\%. En algunas revistas, se observó la publicación simultánea en inglés/ español ( $7,3 \%$ de los casos) e inglés/portugués $(3,8 \%)$. Dos revistas publican de manera exclusiva en idioma español (Av. Odontoestomatol., Odontoestomatología) y cuatro en inglés (Braz. Dent. J., Braz. J. Oral Sci., Braz. Oral Res., S. Afr. Dent. J.). Siete revistas publican en Inglés y español (Int. J. Odontostomat., Rev. Clin. Periodoncia Implantol. Rehabil. Oral, CES Odontol., Rev. Fac. Odontol. Univ. Antioq., Av. Periodoncia. Rev. Esp. Cir. Oral Maxilofac., Rev. Odont. Mex.) y tres revistas en portugués e inglés (Dental Press J. Orthod., J. Appl. Oral Sci., Rev. Odontol. UNESP). La Revista Cubana de Estomatología ha publicado en los tres idioma.

\section{DISCUSIóN}

La odontología, y sus revistas temáticas, han podido integrarse de manera exitosa a los diversos componentes de la metodología SciELO, permitien- 
do la publicación electrónica de ediciones completas de las revistas científicas, la organización de bases de datos bibliográficas y de textos completos, recuperación de textos por su contenido, la preservación de archivos electrónicosy la producción de indicadores estadísticos de uso y impacto de la literatura científica, así como en gran medida, mantener criterios de evaluación de revistas, basado en los estándares internacionales de comunicación científica. Esto refleja una evolución de las revistas odontológicas desde simple publicaciones de casas comerciales hacia una literatura científica independiente y el movimiento hacia un estatus más profesional y basado en la evidencia (Hook, 1985) .

Aunque se identificaron 17 revistas odontológicas en la base SciELO durante el primer semestre del año 2014, sólo algunos países que integran la red poseen revistas del área (Brasil, España, Chile, Colombia, Cuba, México, Sudáfrica y Uruguay), mientras que Argentina, Bolivia, Costa Rica, Paraguay, Perú, Portugal y Venezuela aún no. Con excepción de Argentina y Portugal (con gran cantidad de publicaciones en revistas de otros países), la carencia de revistas odontológicas en dichos países coincide con la baja productividad científica en general y en particular para el área de la odontología.

En América Latina en general, la producción científica en odontología es baja y Brasil es el único país que tiene un buen nivel internacional en esta materia (Gálvez, 2006). Sin embargo, en los últimos años se ha observado una relación directa entre la cantidad de revistas de un país en la base SciELO y el aumento de su producción científica, medida en diferentes índices (SciELO, WoS u Scopus), por lo que no sólo para un país es relevante crear revistas, sino que estas puedan indexarse en bases de calidad. La visibilidad y mejoría en indicadores, debería aumentar más aún, sobretodo por la inclusión de la base SciELO (aunque no en su totalidad aún) a Thomson Reuters y su SciELO Citation Index. Además, resulta interesante la estrategia adoptada por algunas revistas al cambiar su nombre hacia un idioma inglés (i.e. Pesquisa Odontológica Brasileira hacia Brazilian Oral Research) o ampliando su cobertura temática (i.e. Avances en Periodoncia hacia Avances en Periodoncia e Implantología Oral), así como otras modificaciones, todas con el fin de tener una mejor visibilidad nacional e internacional, ha logrado ser una estrategia eficiente.

Pudimos comprobar el gran número y predilec- ción por el idioma inglés al publicar. Este cambio idiomático hacia el inglés por parte de algunas revistas a permitido una mayor visibilidad y lectura anglosajona. Por otra parte, se puede observar una permanencia del español como idioma de publicación; esto que ha sido interpretado como una desventaja en ocasiones, permitiría una adecuación científica a las realidades latinoamericanas, donde no todos tienen un nivel de conocimiento o de lectura del idioma inglés (sobretodo países que no tienen aún revistas en SciELO), pero sí son odontólogos o profesionales que consumen ciencia o ella se encuentra cada vez más a la mano, al publicar en español, les permite consumir la nueva información científica, con una mejor calidad (al estar en la red SciELO) y de manera gratuita por el sistema de acceso abierto que mantiene SciELO.

La base SciELO es un modelo para la publicación electrónica cooperativa de revistas científicas en Internet, especialmente desarrollado para responder a las necesidades de la comunicación científica en los países en desarrollo y particularmente de América Latina y el Caribe, el modelo proporciona una solución eficiente para asegurar la visibilidad y el acceso universal a su literatura científica, contribuyendo para la superación del fenómeno conocido como 'ciencia perdida'. Además, el modelo SciELO contiene procedimientos integrados para la medida del uso y del impacto de las revistas científicas, los cuales son de gran utilidad para la odontología.

Todas las revistas analizadas han aplicado la operación de sitios web de colecciones de revistas electrónicas, a través de sitios nacionales y también temáticos, lo que ha permitido desarrollar alianzas entre los actores nacionales e internacionales de la comunicación científica - autores, editores, instituciones científicas y tecnológicas, agencias de apoyo, universidades, bibliotecas, centros de información científica y tecnológica etc., con el objetivo de diseminar, perfeccionar y mantener el modelo SciELO. La operación de la red SciELO se basa fuertemente en infraestructuras nacionales, lo que contribuye para garantizar su futura sostenibilidad.

Es necesario que los odontólogos conozcan las revistas disponibles en SciELO y sus características como el números de artículos, idioma de publicación, periodicidad entre otros, con el fin de hacer una adecuada selección al momento de enviar su publicación. Este conocimiento toma mayor relevancia cuando se involucran instituciones, universidades o países, ya 
que actualmente, parámetros como la investigación científica son parte de los análisis de desarrollo o aportes financieros para ellos, así como también un parámetro para obtener recursos (postulación a proyectos), postular a becas o generar productos de proyectos con financiamiento o tesis de pre y postgrado.

CANTÍN, M. \& ARAVENA, Y. Dental journals in the SciELO database: a bibliometric overview. Int. J. Odontostomat., 8(2):215-220, 2014.

ABSTRACT: Journals play an important role in the process of scientific communication for various sciences, including dentistry. This communication becomes more important if the journals are indexed on databases of nationally and internationally recognized. The aim of this research was to find, analyze and describe the dental journals indexed in the full record of countries in the SciELO database, until the first half of 2014, in order to inform the dental community status and features major. A descriptive bibliometric methodology was design. Dental journals, abbreviation, date of admission of each journal in the collection, available numbers, quantity and type of articles, and language of publication were identified in the SciELO database. Seventeen dental journals indexed in SciELO database were identified. Between countries, Brazil has more journas, followed by Spain, Chile and Colombia. The first dental journal income in 2000 , and there were 2 new revenue in 2014. There was a direct relationship between the year of income and number of issues published. To date a total of 5,684 articles have been published, mainly original, followed by case reports, literature reviews, editorials and letters to the editor. The language most often used to publish was english (55.2\%), followed by spanish (31.4\%). The portuguese, only accounted for $2.3 \%$. In some journals, simultaneous publication in english/spanish and english/portuguese (3.8\%) was observed. Dentistry, and its thematic journals have been able to successfully integrate the various components of the SciELO methodology. It is necessary that dentists to know available journals in SciELO and their characteristics as numbers, language, periodicity among others, in order to make a proper choice when you submit your publication.

KEYWORDS: science publishing, journals, dentistry, database, SciELO.

\section{REFERENCIAS BIBLIOGRÁFICAS}

Gálvez, M. Publicaciones biomédicas: realidad de Chile y Latinoamérica. Rev. Chil. Radiol., 12(3):113-7, 2006.

Granda-Orive, J. I.; García Río, F.; Gutiérrez Jiménez, T.; Jiménez Ruiz, C. A.; Solano Reina, S. \& Sáez Valls, R. Análisis y evolución de los indicadores bibliométricos de producción y consumo del área de tabaquismo a través de Archivos de Bronco-neumología (período 1970-2000): Comparación con otras áreas neumológicas. Arch. Bronconeumol., 38(II):523-9, 2002.

Hook, S. A. Early dental journalism: a mirror of the development of dentistry as a profession. Bull. Med. Libr. Assoc., 73(4):345-51, 1985.

Pérez, A. C.; Estrada, L. J. M.; Villar, A. F. \& Rebollo, R. M. J. Estudio bibliométrico de los artículos originales de la Revista Española de Salud Pública (1991-2000), parte primera: indicadores generales. Rev. Esp. Salud Pública, 76(6):659-72, 2002.

Tomás-Casterá, V.; Sanz-Valero, J. \& Wanden-Berghe, C. Estudio bibliométrico de la producción científica y de consumo de las revistas sobre nutrición indizadas en la red SciELO (2002 a 2007). Rev. Chil. Nutr., 37(3):3309, 2010.
Dirección para Correspondencia:

Mario Cantín L.

Facultad de Odontología

Universidad de la Frontera

Manuel Montt 112

Casilla 54-D

Temuco, CHILE

Email: mario.cantin@ufrontera.cl

Recibido : 21-06-2014

Aceptado: 01-07-2014 